\begin{tabular}{|c|l|}
\hline Title & Craniofacial Pain and Jaw-muscle A ctivity during Sleep \\
\hline Author(s) & Y achida, W.; Castrillon, E.E.; Baad-Hansen, L.; Jensen, R.; A rima, T.; Tomonaga, A.; O hata, N.; Svensson, P. \\
\hline Citation & $\begin{array}{l}\text { Journal of Dental Research, 91(6), 562-567 } \\
\text { https://doi.org/10.1177/0022034512446340 }\end{array}$ \\
\hline Issue Date & 2012-06-01 \\
\hline Doc URL & http://hdl.handle.net/2115/72855 \\
\hline Type & article (author version) \\
\hline File Information & TMD-TTH-GC-Manuscript-Rev2.pdf \\
\hline
\end{tabular}

Instructions for use 


\section{Craniofacial pain and jaw-muscle activity during sleep}

Wataru Yachida ${ }^{1}$, Eduardo E. Castrillon ${ }^{2}$, Lene Baad-Hansen ${ }^{2}$, Rigmor Jensen ${ }^{3}$ Taro Arima $^{1}$, Akio Tomonaga ${ }^{1}$, Noboru Ohata ${ }^{1}$, Peter Svensson ${ }^{2,4}$

${ }^{1}$ Department of Oral Rehabilitation, Graduate School of Dental Medicine, Hokkaido University, Sapporo, Japan

${ }^{2}$ Clinical Oral Physiology, Department of Dentistry, Aarhus University, Aarhus, Denmark

${ }^{3}$ Danish Headache Centre and Department of Neurology, University of Copenhagen, Glostrup Hospital, Glostrup

${ }^{4}$ Center for Functionally Integrative Neuroscience (CFIN), MindLab, Aarhus University Hospital, Denmark

Correspondence to: Dr. Peter Svensson. Clinical Oral Physiology, Department of Dentistry, Aarhus University, Vennelyst Boulevard 9, DK-8000 Aarhus C, Denmark E-mail: peter.svensson@odontologi.au.dk 


\section{Abstract}

This study aimed to compare the jaw-muscle electromyographic (EMG) activity during sleep in patients with painful $(n=59,36.8 \pm 12.1$ years $)$ or non-painful $(n=56,31.5 \pm 10.8$ years) temporomandibular disorders (TMD), tension-type headache (TTH: $n=30$, $33.3 \pm 9.6$ years $)$, and in healthy controls ( $n=30,26.2 \pm 3.9$ years $)$. All participants used a portable single-channel EMG device (GrindCare ${ }^{\mathrm{TM}}$, MedotechA/S) for one week. There was no significant difference in EMG activity between painful TMD (23.9 \pm 18.0$)$ and non-painful TMD $(20.7 \pm 14.8)$, or between TTH $(20.8 \pm 15.0)$ and healthy subjects $(15.2 \pm 11.6, \mathrm{P}>.050)$. There were, however, correlations between EMG activity and number of painful muscles $(\mathrm{r}=0.188 ; \mathrm{P}=.044)$, characteristic pain intensity $(\mathrm{r}=0.187$; $\mathrm{P}=.046)$, McGill Pain Questionnaire $(\mathrm{r}=0.251 ; \mathrm{P}=.008)$ and depression scores $(\mathrm{r}=0.291$; $\mathrm{P}=.002$ ). Subjects with painful conditions had significantly higher night-to-night variability compared with pain-free subjects $(\mathrm{P}<.050)$. Overall, there seems to be no major differences between different craniofacial pain conditions and healthy controls in jaw-muscle EMG activity recorded with a single-channel EMG device during sleep. However, some associations may exist between the level of EMG activity and various parameters of craniofacial pain. Further studies are warranted.

Key words: myofascial pain, sleep bruxism, portable electromyographic device, 
temporomandibular disorders, tension-type headache, jaw-muscle activity. 


\section{Introduction}

Temporomandibular disorders (TMD) and headache are frequent craniofacial pain conditions in the general population (Jensen and Stovner, 2008; LeResche, 1997). Tension-type headache (TTH) is the most common primary headache (Scher et al., 1998). Sleep bruxism (SB) has been considered to be a risk factor for both TMD and TTH (Baba et al., 2005; Bailey, 1990; Rossetti et al., 2008; Vendrame et al., 2008) and is defined as a sleep-related movement disorder characterized by grinding/clenching of the teeth during sleep and associated with excessive arousal responses (AASM, 2005).

Portable single channel electromyograhic (EMG) recordings have been used to measure SB activity because this method enables multiple night recording in subjects' homes at relatively low costs (Pierce and Gale, 1988; Rugh and Solberg, 1975). In order to understand SB and its potential consequences on oral function and health, a better description of jaw-muscle activity in different craniofacial pain conditions will be needed. Some studies reported the characteristics of jaw-muscle EMG activity in subjects with SB and TMD symptoms (Camparis et al., 2006; van Selms et al., 2008). However, the relationship between $\mathrm{SB}$ and $\mathrm{TMD} / \mathrm{TTH}$ is still controversial and remains unclear (Manfredini and Lobbezoo, 2010; Svensson et al., 2008; Vendrame et al., 2008). Further, some researchers have reported a substantial night-to-night variability of SB 
activity during polysomnography (PSG) (Lavigne et al., 2001; Van Der Zaag et al., 2008). Thus, long-term (multiple nights) recordings of jaw-muscle activity in both painful conditions and healthy controls are necessary to assess the dynamics of SB activity.

The main aim of this study was to compare the jaw-muscle EMG activity during sleep in patients with or without painful TMD, TTH and in healthy controls. We also aimed to explore the correlation between EMG activity and clinical parameters related to physical status, quality of pain and psychological status. Finally, we tested the night-to-night variability of EMG activity during sleep between the different craniofacial pain conditions. 


\section{Materials and Methods}

\section{Subjects}

A total of 115 participants; 39 men (mean age \pm SD: $36.8 \pm 14.0$ years) and 76 women $(32.8 \pm 10.2$ years $)$ participated in this study. All participants were generally healthy and more than 18 years old. Exclusion criteria were current illness; history of neurologic or psychiatric disorders; sleep disorders (e.g. snoring, sleep apnea, and periodic limb movement); use of prescription medicine or drugs; smoking, alcohol abuse and addiction to coffee; electrode gel allergy; simultaneous participation in another trial with medicine or in trials of medical devices; and user of pace maker. The study was approved by the local ethics committee and conducted in accordance with the Helsinki Declaration. Written informed consent was obtained from all participants.

We recruited the following types of participants: 30 healthy subjects (16 men: $25.3 \pm 4.1$ years and 14 women: $27.2 \pm 3.6$ years) who were among students and staff at Aarhus University, Denmark; 55 self-reported SB subjects (16 men: $46.4 \pm 14.6$ years and 39 women: $36.0 \pm 11.5$ years) who were asked by flyers and newspaper advertisement and from patients at the Clinical Oral Physiology, Department of Dentistry, Aarhus University, Aarhus, Denmark, and 30 TTH patients (seven men: 41.1 \pm 7.4 years and 23 women: $31.0 \pm 9.0$ years) from the Danish Headache Center, 
Glostrup Hospital, Denmark.

All healthy subjects were without TMD in accordance with the Research Diagnostic Criteria for TMD (RDC/TMD) (Dworkin and LeResche, 1992). Furthermore, we examined healthy subjects for clinical signs or symptoms related to SB, 1) self-report or reported by a bed partner of tooth-grinding or clenching habits during sleep; 2) jaw-muscle fatigue/pain upon awakening; 3) masseter muscle hypertrophy on voluntary contraction (Lavigne et al., 2008); 4) moderate to severe hyperkeratosis of cheeks/lips/tongue; 5) advanced tooth wear ( $\geq$ grade 1c) (Lobbezoo and Naeije, 2001; Wetselaar et al., 2009); 6) loss of cuspid protection; and 7) frequent non-iatrogenic/non-material related fractures and failures of teeth/restorations/implants. All healthy subjects did not report 1) or 2) and had less than two of the other criteria.

Self-reported SB subjects answered "yes" in RDC/TMD questionnaire 15c which is about self-awareness of SB ("Have you been told, or do you notice that you grind your teeth or clench your jaw while sleeping at night?”).

TTH patients were diagnosed as frequent or chronic TTH according to the diagnostic criteria of the second edition of the International Classification of Headache Disorders (ICHD-II) (The International Classification of Headache Disorders: 2nd edition, 2004) using headache diaries in addition to the RDC/TMD examination and 
questionnaire.

\section{Study design}

On the first day, all participants were asked to fill out the Danish RDC/TMD questionnaire (http://www.rdc-tmdinternational.org) and a Danish version of the McGill Pain Questionnaire (MPQ) (Drewes et al., 1993). The RDC/TMD clinical examination was also performed by standardized examiners (WY, EC, LBH, PS.) who had been calibrated for RDC/TMD and the details of training is showed in the website of International RDC/TMD Consortium (http://www.rdc-tmdinternational.org). Each participant was examined by one examiner. Six self-reported SB subjects did not fill out the MPQ. All participants used a portable EMG device (GrindCare ${ }^{\mathrm{TM}}$, MedotechA/S, Herlev, Denmark) during sleep for at least four nights during one week to measure jaw-muscle activity.

\section{EMG recordings}

The GrindCare $3^{\mathrm{TM}}$ has a single electrode assembly, with three electrode contacts. The electrode was placed at the anterior temporalis muscle, which generally will provide the same information as EMG recordings from the masseter muscle during sleep (Koyano 
et al., 2008). The EMG activity was amplified (x 800) and filtered $(250 \mathrm{~Hz}-610 \mathrm{~Hz})$ in the device and further analyzed for events of EMG activity, using the Signal Recognition Algorithm described in Jadidi et al. (2008). The algorithm is based on a Fast Fourier Transformation analysis and threshold comparison. To determine the individual parameters, the following set-up procedure was performed every night: Subjects were asked to relax their jaw muscle for $10 \mathrm{sec}$. Then subjects made a grimace for $10 \mathrm{sec}$ and then clenched their teeth around $60 \%$ of the maximum voluntary contraction for $10 \mathrm{sec}$. These procedures were carefully instructed by the examiners and trained together with the participants. The algorithm counts an event when the EMG activity exceeds the signal level at rest, plus $20 \%$ of the maximum EMG level during the $60 \%$ contraction. After each detection, the system waits for one second, and if the activity is still, or again, above threshold, a new event is counted. The total number of EMG events, the number of EMG events per hour and the number of measurement hours were registered. After one week of measurement had finished, the data were transferred and saved in a PC using commercial software (GrindCare Manager, MedotechA/S, Herlev, Denmark).

\section{Clinical parameters}


Four variables from the RDC/TMD questionnaire and clinical examination; maximum pain-free jaw opening, number of painful muscles (0-20), characteristic pain intensity (CPI: 0-100) (Dworkin, 1990) and depression score were selected as secondary outcome clinical parameters. The MPQ was also used to assess the sensory, affective, evaluative, and miscellaneous dimension of pain (Melzack, 1975). The total pain rating index (PRI) was used in the analyses.

\section{Data analysis}

All participants were divided into painful and non-painful TMD groups according to the RDC/TMD (Fig.1A). Painful TMD subjects were diagnosed as myofascial pain (RDC/TMD group1ab)/arthralgia (RDC/TMD group3a). Non-painful TMD subjects could have a diagnosis of disc displacement (RDC/TMD group2abc) but without any pain or a diagnosis of osteoarthrosis (RDC/TMD group3c). These groups were also split into with or without self-reported SB (Fig.1B). Then, we compared the EMG data in each group to see the influence of painful TMD/self-reported SB on EMG activity. Further, we compared EMG activity between TTH and healthy subjects. Then, we divided TTH subjects into groups with or without self-reported SB and compared the EMG activity between groups. The coefficient of variation from the multiple night 
recordings (CV: $\mathrm{SD} /$ mean) was calculated for all individuals in each group to examine the night-to-night variability in EMG activity.

Finally, we analyzed the correlation between EMG activity and some clinical variables derived from the RDC/TMD questionnaire and clinical examination and MPQ.

\section{Statistics}

The data are presented as mean \pm standard deviation (SD). We used non-paired $t$-tests to analyze EMG data and CV between with and without painful TMD groups. Two-way analysis of variance (ANOVA) was used to test the effect of painful TMD and self-reported SB on EMG activity and $\mathrm{CV}$. When appropriate, post-hoc tests were performed with Tukey tests (with correction for multiple comparisons). We also used non-paired $t$-tests to analyze EMG activity and CV between TTH and healthy subjects and between TTH with and without self-reported SB. Spearman correlation tests were used to test the association between EMG activity and clinical parameters. The level of significance was set at $\mathrm{P}<.050$. 


\section{Results}

\section{Between group differences}

There was no significant difference in EMG activity between painful and non-painful TMD groups (painful: $23.9 \pm 18.0$, non-painful: $20.7 \pm 14.8, t$-test, $\mathrm{P}=.307$, Fig. $2 \mathrm{~A}$ ). There was no significant effect of painful TMD ( $\mathrm{F}=0.023, \mathrm{P}=.879)$, but a significant effect of self-reported SB $(\mathrm{F}=9.225, \mathrm{P}<.050)$, with no significant interaction between factors $(\mathrm{F}=0.153, \mathrm{P}=.697$, Fig.2B). Self-reported $\mathrm{SB}$ subjects had significantly higher EMG activity compared with non- SB subjects (Tukey, $\mathrm{P}<.050$, Fig.2B). There was no significant difference between TTH patients $(20.8 \pm 15.0)$ and healthy subjects $(15.2 \pm$ 11.6) in EMG activity ( $t$-test, $\mathrm{P}=.108$, Fig.2C) or between TTH patients with and without self-reported SB (t-test, $\mathrm{P}=.294$, Fig.2D). All participants had measurement times $>6$ hours.

\section{Night-to-night variability}

Painful TMD patients had significantly higher CV compared with non-painful TMD subjects ( $t$-test, $\mathrm{P}<.050$, Fig.3A). There was a significant effect of painful TMD $(\mathrm{F}=7.779, \mathrm{P}<.050)$, but no significant effect of self-reported $\mathrm{SB}(\mathrm{F}=2.032, \mathrm{P}=.157)$, with no significant interaction between factors $(\mathrm{F}=2.515, \mathrm{P}=.116$, Fig.3B). There were 
significant difference in CV between painful TMD and non-painful TMD (Tukey, $\mathrm{P}<.050$, Fig. 3B). TTH patients had significantly higher CV compared with healthy subjects ( $t$-test, $\mathrm{P}<.050$, Fig.3C). There was no significant difference between TTH with and without self-reported SB ( $t$-test, $\mathrm{P}=.256$, Fig.3D).

\section{Correlation between EMG activity and clinical parameters}

There were significant positive correlations between the number of EMG events per hour sleep and number of painful muscles $(\mathrm{r}=0.188, \mathrm{P}=.044)$, CPI $(\mathrm{r}=0.187, \mathrm{P}=.046)$, depression scores $(\mathrm{r}=0.291, \mathrm{P}=.002)$ and MPQ ( $\mathrm{r}=0.251, \mathrm{P}=.008$, Fig. 4). 


\section{Discussion}

The main finding in this study was the lack of significant differences in EMG activity during sleep between patients with craniofacial pain conditions (TMD, TTH) and healthy control subjects. In a previous study, Rossetti et al. (2008) reported that rhythmic masticatory muscle activity (RMMA) during sleep was weakly associated with myofascial pain by PSG recordings $(n=60)$ which was regarded as the gold standard for assessing SB. In contrast, van Selms et al. (2008) reported no relationship between masticatory muscle activity during sleep and muscle pain using an ambulatory EMG device $(n=4)$. In this study, there was no significant difference in EMG activity between subjects with painful conditions and pain-free subjects. Further, we did an additional analysis comparing the EMG activity between painful TMD/TTH $(n=63)$ and non-painful groups (without TMD pain and TTH; n=52) which did not indicate any significant differences between the groups. However, there was a significant correlation between EMG activity and CPI, and painful TMD and TTH patients had higher CV compared with pain-free subjects. Perhaps certain types or characteristics of muscle activities (intensity, duration, frequency, direction of forces) may be associated with craniofacial pain conditions, and painful conditions may increase the variability. The causal relationship between SB and painful conditions is still a matter of discussion 
(Svensson et al., 2008) and the present study design does not allow any definitive conclusions on the causal relationships between SB and craniofacial pain. We showed, however, that jaw-muscle EMG activity during sleep cannot be discriminated between different craniofacial pain conditions. Another important result was that EMG activity during sleep was strongly influenced by self-reports of SB in the entire study group but not specifically in TTH patients. TTH could reduce the reliability of self-awareness of SB. The reliability of self-reports of SB is generally low (Koyano et al., 2008) and may have influenced our findings. Further research would be needed to reveal to what extent painful conditions affect self-reports of SB.

Lavigne et al. (2001) reported that the CV was $25.3 \%$ for the number of EMG episodes per hour of sleep in subjects with SB using PSG recordings. Our study showed higher $\mathrm{CV}$ in all groups compared with previous data. This difference could be due to the different way of measurement and analyses but strongly indicates that there is a significant and substantial night-to-night variability in EMG recordings which needs to be taken into consideration when evaluating SB.

There were weak, but significant correlations between EMG activity and depression and MPQ scores. EMG activity during sleep may be much more complex and rather related to psychological/psychosocial status than to physical status. However, 
Manfredini and Lobbezoo (2009) concluded that there seems to be no evidence to relate SB with psychosocial factors. The role of psychological/psychosocial factors in the etiology of bruxism would be one of the most debated issues, so we also need more studies to improve our understanding of the relationship between SB and psychological/psychosocial factors. The present finding indicates that a single channel EMG device may be helpful in this respect.

This study has some limitations. The recording term was not same in all participants: some subjects forgot using the device or the measurement was failed. We could not re-record the measurement because of practical issues, large samples with multiple night recordings. Further, ambulatory recording system did not allow researcher-controlled environment, so the reliability of the data would be lower compared with PSG study. It is important to realize these limitations in ambulatory recording study.

In conclusion, there were no major differences between patients with different craniofacial pain conditions and healthy controls in jaw-muscle EMG activity recorded with a single-channel EMG device during sleep. However, some associations may exist between the level of EMG activity and various parameters of craniofacial pain. Further studies are warranted to enhance the understanding of SB and potential consequences 
such as painful conditions. 


\section{Acknowledgments}

We thank N. Caspersen, physical therapist at the Danish Headache Center, for her participation in the recruitment of TTH patients to the study and M. Haugland, who works for MedotechA/S, for his advices to the technical aspects of the study. The study was, in part, supported by MedotechA/S.

\section{Conflicts of interest statement}

P. Svensson is a paid consultant for MedotechA/S, R. Jensen is a member of the advisory board of the company and E. Castrillon's post-doc fellowship was partially funded by the company. Neither of the authors have stocks in MedotechA/S. 


\section{References}

American Academy of Sleep Medicine (2005). International classification of sleep disorders. 2nd ed. Diagnostic and coding manual. Westchester, IL:American Academy of Sleep Medicine.

Baba K, Haketa T, Sasaki Y, Ohyama T, Clark GT (2005). Association between masseter muscle activity levels recorded during sleep and signs and symptoms of temporomandibular disorders in healthy young adults. J Orofac Pain 19:226-231.

Bailey DR (1990). Tension headache and bruxism in the sleep disordered patient. Cranio 8:174-182.

Camparis CM, Formigoni G, Teixeira MJ, Bittencourt LR, Tufik S, de Siqueira JT (2006). Sleep bruxism and temporomandibular disorder: Clinical and polysomnographic evaluation. Arch Oral Biol 51:721-728.

Clark GT, Rugh JD, Handelman SL (1980). Nocturnal masseter muscle activity and urinary catecholamine levels in bruxers. J Dent Res 59:1571-1576.

Drewes AM, Helweg-Larsen S, Petersen P, Brennum J, Andreasen A, Poulsen LH et al. (1993). McGill Pain Questionnaire translated into Danish: experimental and clinical findings. Clin J Pain 9:80-87.

Dworkin SF, LeResche L (1992). Research diagnostic criteria for temporomandibular disorders: review, criteria, examinations and specifications, critique. J Craniomandib Disord 6:301-355.

Dworkin SF, Von Korff, M., Whitney, C.W., Le Resche, L., Dicker, B.G. and Barlow, W. (1990). Measurement of characteristic pain intensity in field research. Pain Suppl 5(S290).

Gallo LM, Gross SS, Palla S (1999). Nocturnal masseter EMG activity of healthy subjects in a natural environment. $J$ Dent Res 78:1436-1444.

The International Classification of Headache Disorders: 2nd edition. (2004). 
Cephalalgia 24 Suppl 1(9-160).

Jadidi F, Castrillon E, Svensson P (2008). Effect of conditioning electrical stimuli on temporalis electromyographic activity during sleep. J Oral Rehabil 35:171-183.

Jensen R, Stovner LJ (2008). Epidemiology and comorbidity of headache. Lancet Neurol 7:354-361.

Koyano K, Tsukiyama Y, Ichiki R, Kuwata T (2008). Assessment of bruxism in the clinic. J Oral Rehabil 35:495-508.

Lavigne GJ, Guitard F, Rompre PH, Montplaisir JY (2001). Variability in sleep bruxism activity over time. J Sleep Res 10:237-244.

Lavigne GJ, Khoury S, Abe S, Yamaguchi T, Raphael K (2008). Bruxism physiology and pathology: an overview for clinicians. J Oral Rehabil 35:476-494.

LeResche L (1997). Epidemiology of temporomandibular disorders: implications for the investigation of etiologic factors. Crit Rev Oral Biol Med 8:291-305.

Lobbezoo F, Naeije M (2001). A reliability study of clinical tooth wear measurements. $J$ Prosthet Dent 86:597-602.

Manfredini D, Lobbezoo F (2009). Role of psychosocial factors in the etiology of bruxism. J Orofac Pain 23:153-166.

Manfredini D, Lobbezoo F (2010). Relationship between bruxism and temporomandibular disorders: a systematic review of literature from 1998 to 2008. Oral Surg Oral Med Oral Pathol Oral Radiol Endod 109:e26-50.

Melzack R (1975). The McGill Pain Questionnaire: major properties and scoring methods. Pain 1:277-299.

Pierce CJ, Gale EN (1988). A comparison of different treatments for nocturnal bruxism. J Dent Res 67:597-601. 
Rossetti LM, Pereira de Araujo Cdos R, Rossetti PH, Conti PC (2008). Association between rhythmic masticatory muscle activity during sleep and masticatory myofascial pain: a polysomnographic study. J Orofac Pain 22:190-200.

Rugh JD, Solberg WK (1975). Electromyographic studies of bruxist behavior before and during treatment. J Calif Dent Assoc 3:56-59.

Scher AI, Stewart WF, Liberman J, Lipton RB (1998). Prevalence of frequent headache in a population sample. Headache 38:497-506.

Svensson P, Jadidi F, Arima T, Baad-Hansen L, Sessle BJ (2008). Relationships between craniofacial pain and bruxism. J Oral Rehabil 35:524-547.

Van Der Zaag J, Lobbezoo F, Visscher CM, Hamburger HL, Naeije M (2008). Time-variant nature of sleep bruxism outcome variables using ambulatory polysomnography: implications for recognition and therapy evaluation. J Oral Rehabil 35:577-584.

van Selms MK, Lobbezoo F, Visscher CM, Naeije M (2008). Myofascial temporomandibular disorder pain, parafunctions and psychological stress. J Oral Rehabil 35:45-52.

Vendrame M, Kaleyias J, Valencia I, Legido A, Kothare SV (2008). Polysomnographic findings in children with headaches. Pediatr Neurol 39:6-11.

Wetselaar P, Lobbezoo F, Koutris M, Visscher CM, Naeije M (2009). Reliability of an occlusal and nonocclusal tooth wear grading system: clinical use versus dental cast assessment. Int J Prosthodont 22:388-390. 
Figure legends

Fig. 1

Flowchart of grouping of all participants in analysis of EMG data and CV. (A) is painful

TMD (pTMD (+)) and non-painful TMD (pTMD (-)) groups. (B) is painful TMD with

or without self-reported SB (pTMD (+) and SB (+) or (-)) and non-painful TMD with

and without self-reported group (pTMD (-) and SB (+) or (-)).

Fig. 2

Comparison of the EMG data (the number of EMG events per hour of sleep) between

different groups. (A) Painful TMD (pTMD (+)) and non-painful TMD (pTMD (-))

groups, $(\mathrm{B}) *$ indicates a significant effect of self-reported SB (ANOVA: $\mathrm{P}<.050)$ and ${ }^{\#}$ indicates a significant difference between with and without self-reported SB (Tukey: P

$<.050$ ). (C) TTH and healthy groups, (D) TTH with and without self-reported SB

groups. Mean values and SD.

Fig. 3

Comparison of $\mathrm{CV}$ values between different groups. (A) * indicates significant 
difference between painful TMD (pTMD $(+))$ and non-painful TMD $(\mathrm{pTMD}(-))$ groups $(t$-test: $\mathrm{P}<.050),(\mathrm{B}) *$ indicates significant effect of painful TMD $($ ANOVA: $\mathrm{P}<.050)$ and ${ }^{\#}$ indicates significant difference between painful TMD and non-painful TMD groups (Tukey: $\mathrm{P}<.050)$. (C) * indicates significant difference between TTH and healthy groups ( $t$-test: $\mathrm{P}<.050)(\mathrm{D}) \mathrm{TTH}$ with and without self-reported SB groups. Mean values and SD.

Fig. 4

Correlation between EMG data (the number of EMG events per hour of sleep) and clinical parameters. (A) Maximum pain-free jaw opening, (B) Number of painful muscles, (C) CPI score, (D) Depression score, (E) MPQ score. Correlation coefficient $(\mathrm{r})$ is from Spearman correlation tests. 
Fig. 1




Fig. 2
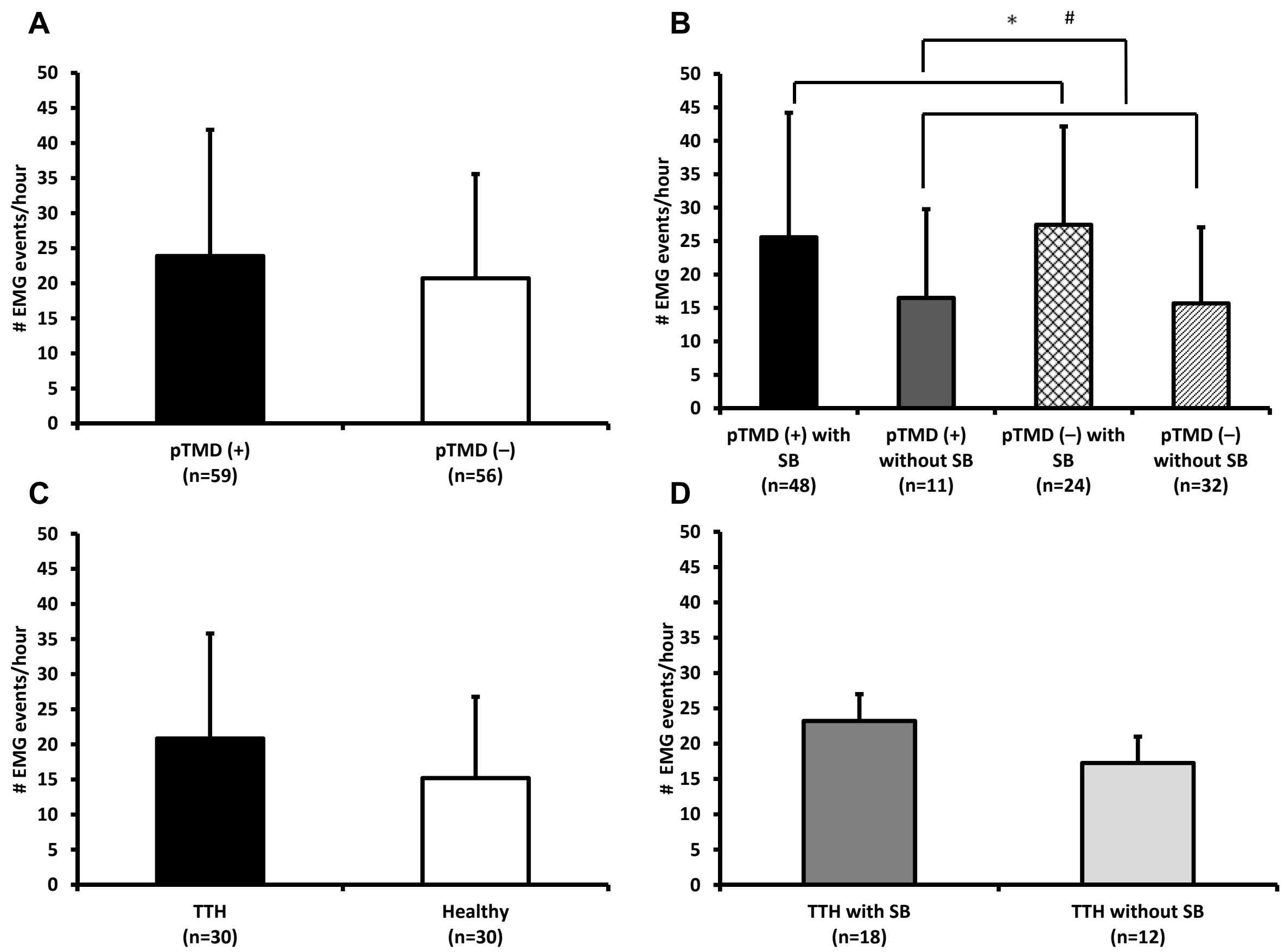
Fig. 3

\section{A}
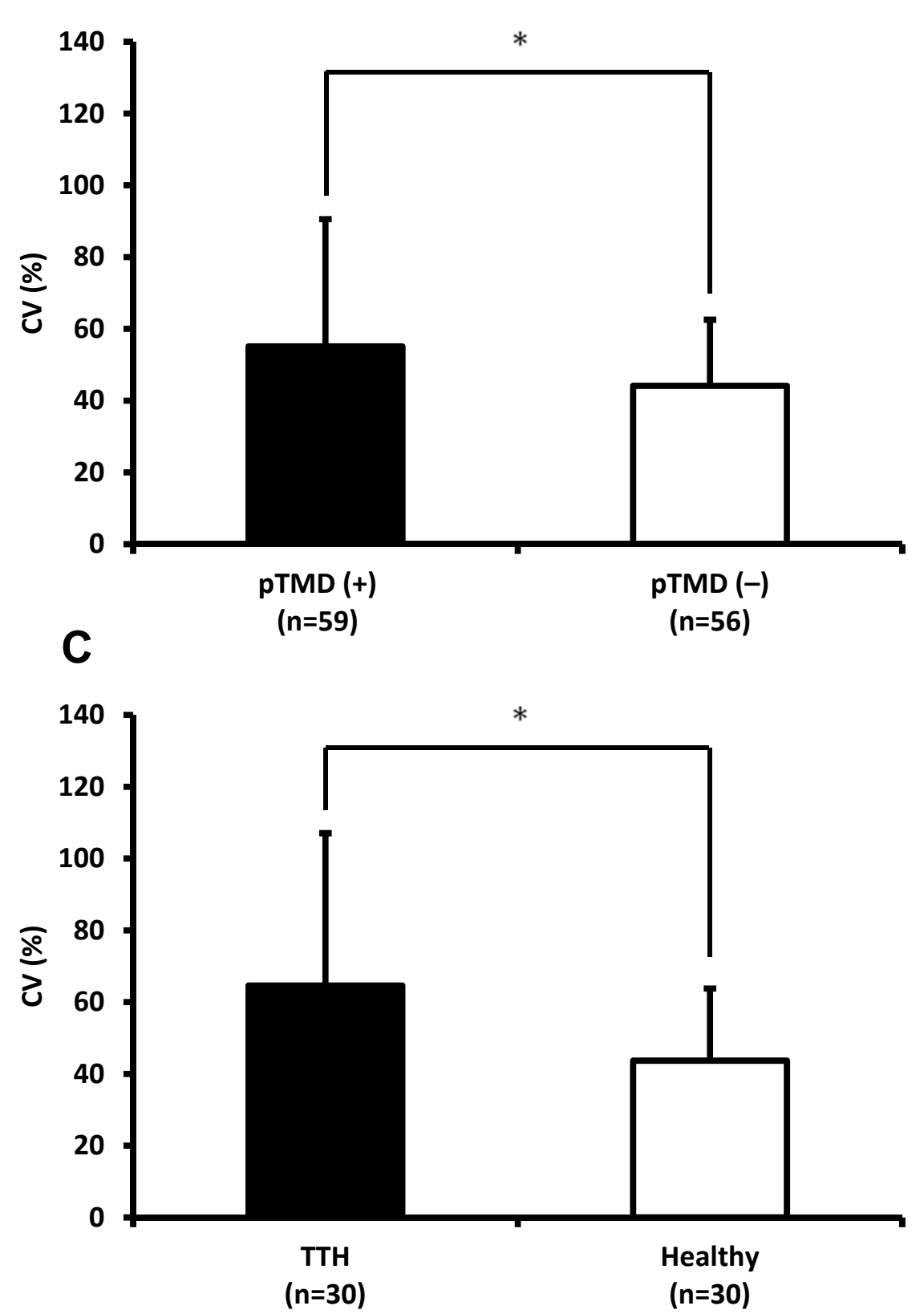

B
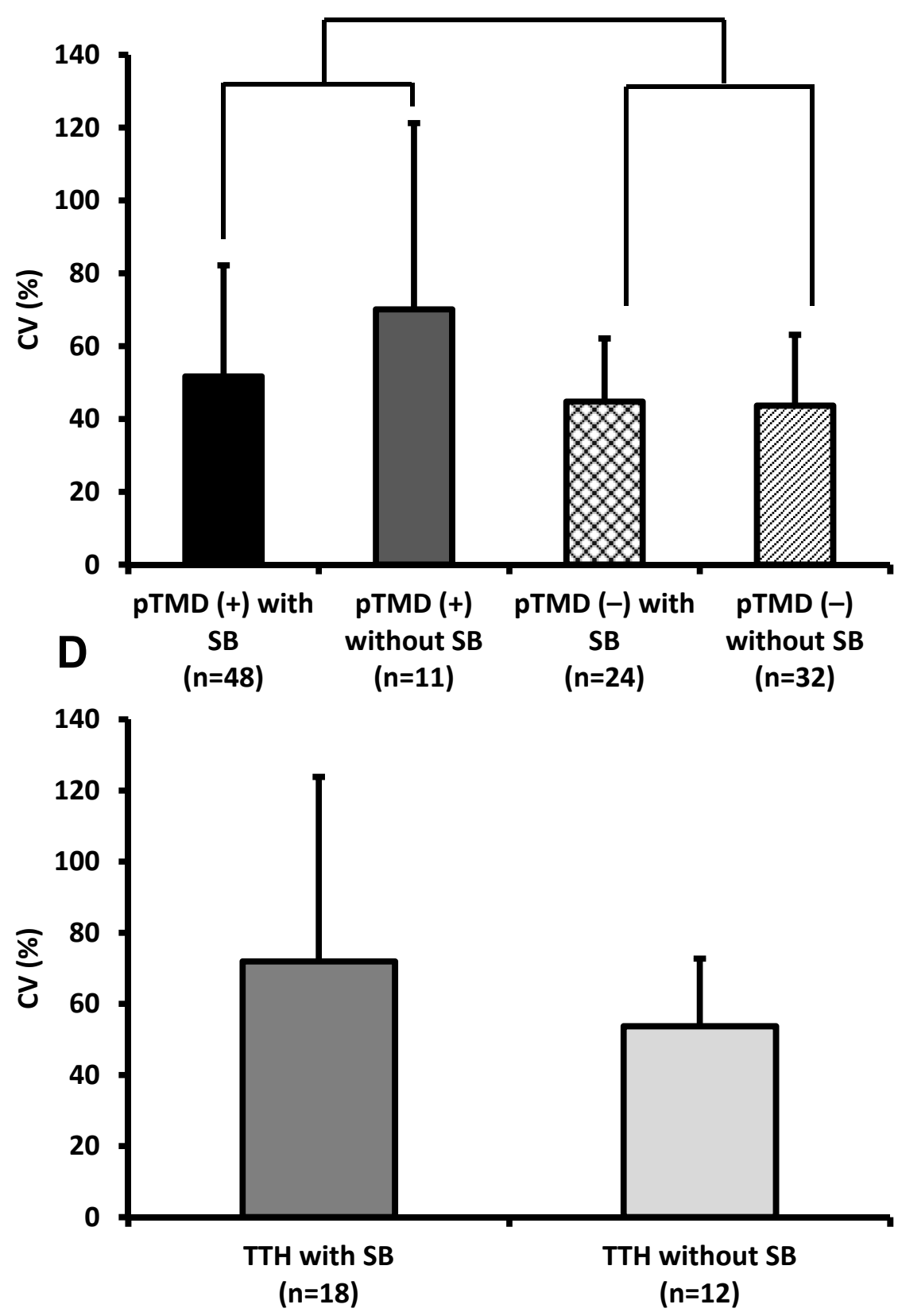
Fig. 4
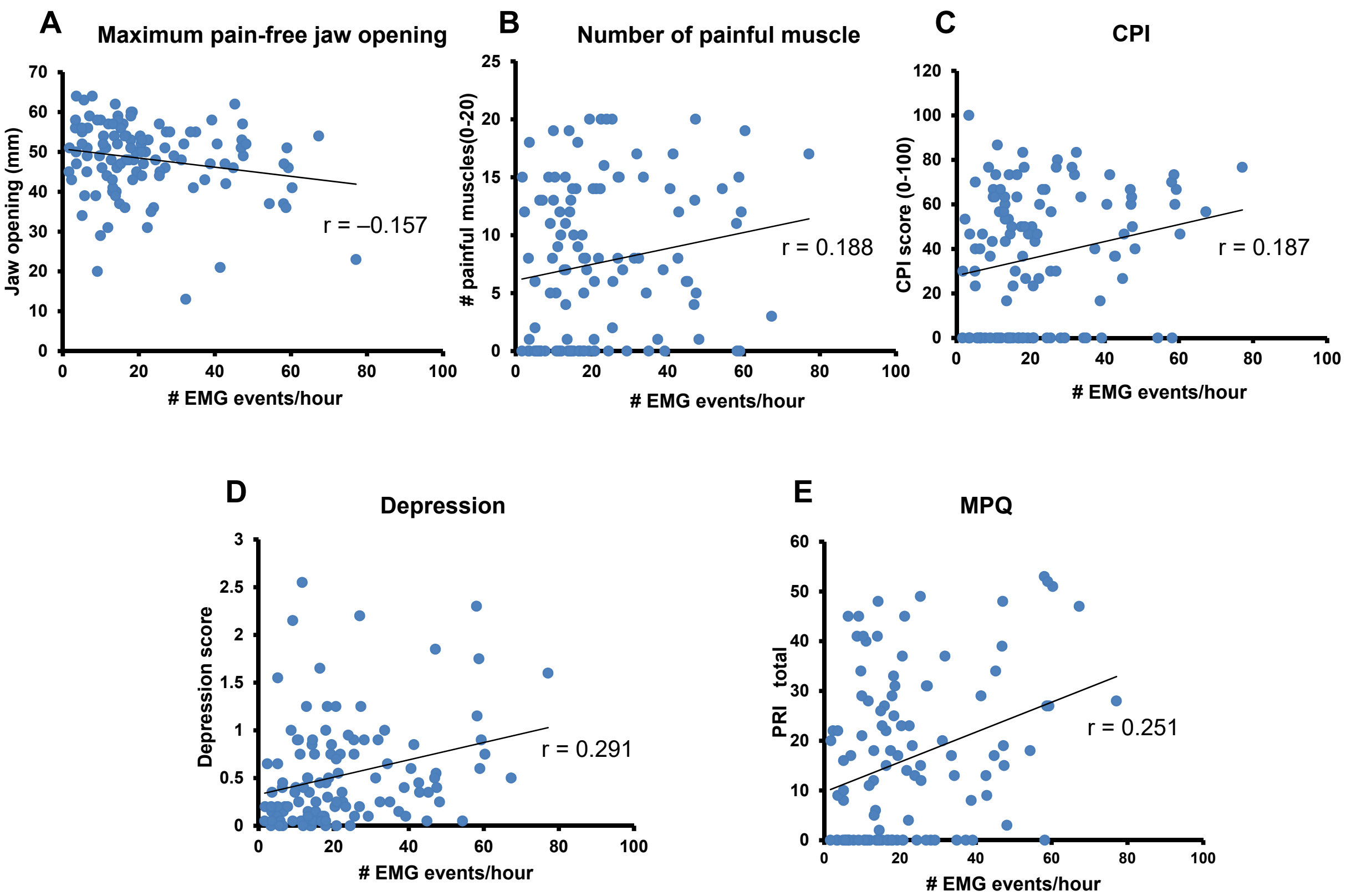